\title{
ON CERTAIN MATRICES AND THEIR DETERMINANTS*
}

\author{
BY W. E. ROTH
}

1. Introduction. It is well known that certain determinants, $\left|c_{i j}\right|,(i, j=1,2, \cdots, r)$, are expressible as the products of $r$ linear factors of the form $a_{i 1} \omega_{i 1}+a_{i 2} \omega_{i 2}+\cdots+a_{i r} \omega_{i r}, \quad(i=1$, $2, \cdots, r)$, where $a_{i j}$ are rational functions of the elements, $c_{1 j}$, in the first row of $\left|c_{i j}\right|$, and where $\omega_{i j}$ are functions of certain roots of unity and depend only on the relations existing between the elements of succeeding rows and those of the first row of the given determinants. Cyclic determinants and some related types which have this property are well known. $\dagger$

In the present paper a generalization of these results is carried forward in two directions; first, we replace the scalar elements in the array of the given determinant by square matrices of order $n$; and second, we permit succeeding rows of the array to be other than permutations of the matrices occurring say in the first row of the given determinant. Certain special types, which we here generalize, have been studied by Puchta, Noether, Baltzer, Drude, Burnside, Scorza, and others. $\ddagger$

* Presented to the Society, April 9, 1937.

† Pascal, Die Determinanten, \$\$20-21. Gegenbauer, Über eine specielle symmetrische Determinante, Sitzungsberichte, Akademie der Wissenschaften, Mathematische-Naturwissenschaftliche Klasse, vol. 82, II-III (1880), pp. 938-942. Burnside, On a property of certain determinants, Messenger of Mathematics, vol. 23 (1894), pp. 112-114.

$\ddagger$ Puchta, Ein Determinantensatz und seine Umkehrung, Denkschriften der Wiener Akademie, vol. 38, $2^{\text {te }}$ Abth. (1878), pp. 215-221; Ein neuer Satz aus der Theorie der Determinanten, ibid., vol. 44, 2 $2^{\text {te }}$ Abth. (1882), pp. 227-282.

Noether, Zur Theorie der Thetafunctionen, Mathematische Annalen, vol. 16 (1880), pp. 322-325; Notiz über eine Classe symmetrischer Determinanten, ibid., pp. 551-555.

Baltzer, Ueber einen Satz aus der Determinantentheorie, Nachrichten, Königlich Gesellschaft der Wissenschaften, Göttingen, 1887, pp. 389-391.

Drude, Ein Satz aus der Determinantentheorie, ibid., pp. 118-122.

Burnside, loc. ciț.

Scorza, Sopra una certa classe di determinanti e sulla forma Hermitiane, Giornale di Matematica, vol. 51 (1913), pp. 335-342. 
2. Direct Product Matrices. If $A$ and $B$ are $n \times n$ matrices and if

$$
U=\left(u_{i j}\right), \quad V=\left(v_{i j}\right), \quad(i, j=1,2, \cdots, r),
$$

are $r \times r$ matrices whose elements $u_{i j}$ and $v_{i j}$ are commutative with those of $A$ and of $B$, then the $n r \times n r$ matrix

$$
\langle A\rangle U=U\langle A\rangle=\left(u_{i j} A\right)=\left(A u_{i j}\right),
$$

is the direct product of $A$ and $U$, and it readily follows that

$$
\langle A\rangle U \cdot\langle B\rangle V=\langle A B\rangle(U V)=(U V)\langle A B\rangle .
$$

Moreover, the matrices $\langle A\rangle U$ and $A\langle U\rangle$ are equivalent in that their characteristic matrices have identical elementary divisors.*

3. The Matrix $\left\langle A_{0}\right\rangle I_{r}+\left\langle A_{1}\right\rangle U+\cdots+\left\langle A_{r-1}\right\rangle U^{r-1}$. We shall assume that the elements of the $n \times n$ matrices, $A_{i}, \quad(i=0$, $1, \cdots, r-1$ ), belong to the field $F$, and that the $r \times r$ matrix $U$, likewise with elements in $F$, is such that $U-\lambda I$ has the elementary divisors $\left(\theta_{i}-\lambda\right)^{r_{i}},(i=1,2, \cdots, s)$, where $\theta_{i},(i=1,2, \cdots, r)$, are not necessarily in $F$. Let the non-singular matrix, $Q$, with elements in $F$ extended by the adjunction of the roots of $|U-\lambda I|=\phi(\lambda)=0$, be such that

$$
Q U Q^{-1}=U_{1}+U_{2}+\cdots+U_{s},
$$

where

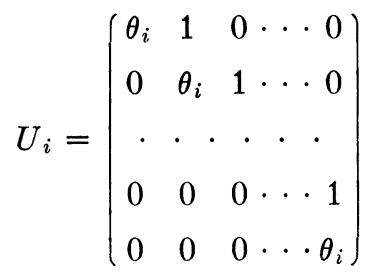

is an $r_{i} \times r_{i}$ matrix and $\sum_{i=1}^{s} r_{i}=r$. For convenience, we shall designate as $B(U)$ the $n r \times n r$ matrix discussed in this section:

$$
B(U)=\left\langle A_{0}\right\rangle I_{r}+\left\langle A_{1}\right\rangle U+\cdots+\left\langle A_{r-1}\right\rangle U^{r-1},
$$

where $I_{r}$ is the unit matrix of order $r$. Hence according to (1) and (2) we have

* For further details on the algebra of direct product matrices see MacDuffee, The Theory of Matrices, Springer, 1933, Chapter VII; and Roth, On direct product matrices, this Bulletin, vol. 40 (1934), pp. 461-468. 


$$
\left\langle I_{r}\right\rangle Q \cdot B(U) \cdot\left\langle I_{r}\right\rangle Q^{-1}=B\left(U_{1}\right)+B\left(U_{2}\right)+\cdots+B\left(U_{s}\right),
$$

and

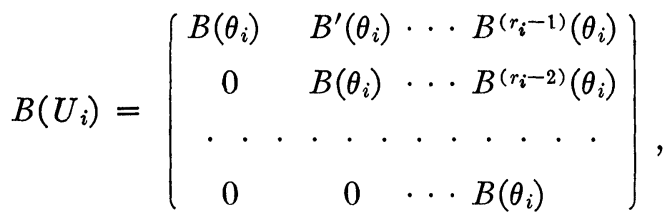

where

$$
B^{(k)}\left(\theta_{i}\right)=C_{k, k} A_{k}+C_{k+1, k} A_{k+1} \theta_{i}+\cdots+C_{r_{i}-1, k} A_{r_{i}-1} \theta^{r_{i}-k-1},
$$

$\left(k=0,1, \cdots, r_{i}-1 ; i=1,2, \cdots, s\right)$, the $C$ 's being binomial coefficients. We have proved the theorem:

THEORE M. If

$$
B(U)=\left\langle A_{0}\right\rangle I_{r}+\left\langle A_{1}\right\rangle U+\cdots+\left\langle A_{r-1}\right\rangle U^{r-1},
$$

where the $A_{i}$ are $n \times n$ matrices with elements in $F$, and if $U$, an $r \times r$ matrix with elements in $F$, is equivalent to the direct sum $U_{1}+U_{2}+\cdots+U_{s}$, where the $U_{i},(i=1,2, \cdots, s)$, are given by $(3)$; then $B(U)$ is equivalent to the direct sum

$$
B\left(U_{1}\right)+B\left(U_{2}\right)+\cdots+B\left(U_{s}\right),
$$

where the $B\left(U_{i}\right),(i=1,2, \cdots, s)$, given by (4) have their elements in $F$ extended by the adjunction of the roots of $|U-\lambda I|=0$.

This theorem, whose proof as given above is almost trivial, has very extensive applications in the theory of determinants. A few of these will be illustrated in the following section.

4. Determinants. The theorem above leads at once to the following :

CoRolla Ry. If $\theta_{i},(i=1,2, \cdots, r)$, are the characteristic roots of $U$, whether distinct or not, then

$$
|B(U)|=\prod_{i=1}^{r}\left|B\left(\theta_{i}\right)\right| *^{*}
$$

* Williamson (The latent roots of a matrix of special type, this Bulletin, vol. 37 (1931), pp. 585-590, Theorem I) and Finan (A theorem on matrices, abstract 41-11-359, this Bulletin) have obtained results closely allied to this corollary. 
That is, the $n r \times n r$ determinant $|B(U)|$ is expressible as the product of $r$ determinants, $\left|B\left(\theta_{i}\right)\right|,(i=1,2, \cdots, r)$, of order $n$. For example, the determinants studied by Baltzer* and by Scorza $\uparrow$ would, according to our notation, be given by

$$
\Delta=\left|\begin{array}{rr}
A_{0} & A_{1} \\
-A_{1} & A_{0}
\end{array}\right|=\left|\left\langle A_{0}\right\rangle I_{2}+\left\langle A_{1}\right\rangle U\right|, \text { where } U=\left(\begin{array}{rr}
0 & 1 \\
-1 & 0
\end{array}\right),
$$

and since $|U-\lambda I|=(i-\lambda)(-i-\lambda)$, we have

$$
\Delta=\left|A_{0}+i A_{1}\right| \cdot\left|A_{0}-i A_{1}\right| .
$$

Hence the given determinant is the sum of two squares provided the elements of $A_{0}$ and $A_{1}$ are real. Also we can conclude that if $A_{0}+i A_{1}$, where $A_{0}$ and $A_{1}$ are real, have a real characteristic root, then $\left\langle A_{0}\right\rangle I_{2}+\left\langle A_{1}\right\rangle U$ above has this root as a multiple characteristic root. $\ddagger$

The cyclic determinant

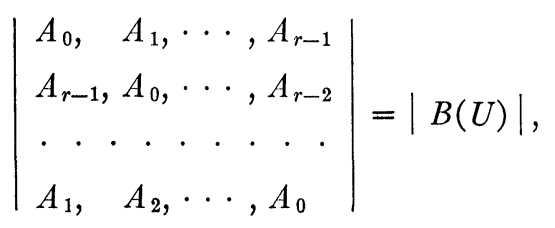

where

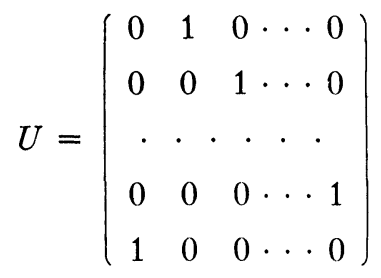

and the $A_{i},(i=0,1, \cdots, r-1)$, are $n \times n$ matrices, is given by the product

$$
\prod_{i=1}^{r}\left|A_{0}+A_{1} \omega^{i}+\cdots+A_{r-1} \omega^{(r-1) i}\right|
$$

* Baltzer, loc. cit.

$\dagger$ Scorza, loc. cit.

$\ddagger$ Wedderburn, Lectures on Matrices, p. 101. 
where $\omega$ is a primitive $r$ th root of unity, that is, a primitive root of $\left|U-\lambda I_{r}\right|=0$.

Each of the factors, $\left|B\left(\theta_{i}\right)\right|,(i=1,2, \cdots, r)$, in (5) is again expressible as the product of $s$ factors, if the $n \times n$ matrices $A_{i}$, $(i=0,1, \cdots, r-1)$, are given by

$$
A_{i}=\left\langle A_{i, 0}\right\rangle I_{s}+\left\langle A_{i, 1}\right\rangle V+\cdots+\left\langle A_{i, s-1}\right\rangle V^{s-1},
$$

where the $A_{i j},(i=0,1, \cdots, r-1 ; j=0,1, \cdots, s-1)$, are $m \times m$ matrices, where $V$ is an $s \times s$ matrix, and $n=m s$. This factorization may be continued still farther under certain conditions which are now obvious.

Matrices of the form given by $B(U)$ above have arisen frequently in the literature, particularly in the theory of algebraic numbers. For example, the $n \times n$ matrix whose elements are algebraic numbers with $\theta$, a root of the equation

$$
a_{0}+a_{1} \lambda+\cdots+a_{r-1} \lambda^{r-1}-\lambda^{r}=0,
$$

as basis, can be written

$$
B(\theta)=A_{0}+A_{1} \theta+\cdots+A_{r-1} \theta^{r-1}
$$

where the $A_{i},(i=0,1, \cdots, r-1)$, are $n \times n$ matrices. Then

$$
\theta B(\theta)=A_{0}^{\prime}+A_{1}^{\prime} \theta+\cdots+A_{r-1}^{\prime} \theta^{r-1},
$$

where

$A_{0}^{\prime}=a_{0} A_{r-1}, \quad A_{i}^{\prime}=A_{i-1}+a_{i} A_{r-1}, \quad(i=1,2, \cdots, r-1)$.

Similarly

$$
\theta^{2} B(\theta)=A_{0}^{\prime \prime}+A_{1}^{\prime \prime} \theta+\cdots+A_{r-1}^{\prime \prime} \theta^{r-1}
$$

where

$A_{0}^{\prime \prime}=a_{0} A_{r-1}^{\prime}, \quad A_{i}^{\prime \prime}=A_{i-1}^{\prime}+a_{i} A_{r-1}^{\prime}, \quad(i=1,2, \cdots, r-1)$,

and so on. Hence the $n r \times n$ matrix

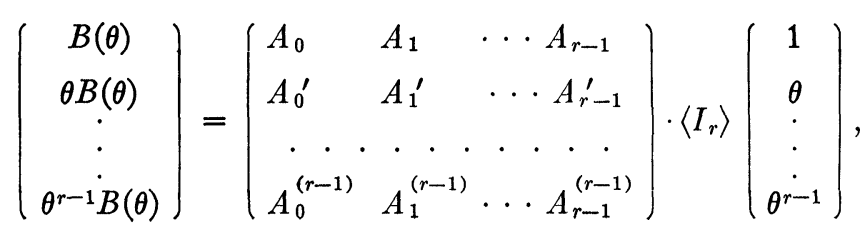




$$
=B(U) \cdot\left\langle I_{r}\right\rangle\left(\begin{array}{c}
1 \\
\theta \\
\cdot \\
\theta^{r-1}
\end{array}\right),
$$

where

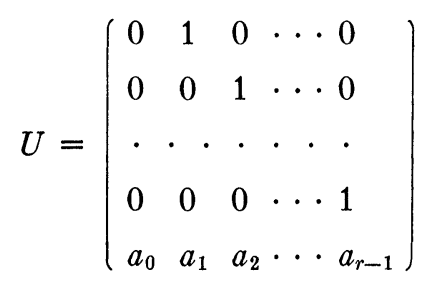

and

$$
B(U)=\left\langle A_{0}\right\rangle I_{r}+\left\langle A_{1}\right\rangle U+\cdots+\left\langle A_{r-1}\right\rangle U^{r-1} .
$$

The $n r \times n r$ matrix $B(U)$ in $F$ corresponds to the $n \times n$ matrix $B(\theta)$, whose elements are algebraic numbers, in the sense of an isomorphism under addition and multiplication.*

UNIVERSITY OF WISCONSIN,

Extension Division, Milwaukee

* The determinant, $B(U)$, may be regarded as the norm of $B(\theta)$. See Dickson, Algebras and their Arithmetics, p. 70. 